\title{
PROPOSTA PARA A CONSTRUÇÃO DE UM AMBIENTE DE INOVAÇÃO PARA REDES DE TELECOMUNICAÇÕES SUAS CONTRIBUIÇÕES PARA A MANUTENÇÃO DO PODER INFORMACIONAL DO ESTADO NA SEGURANÇA PÚBLICA
}

\author{
Luciano Castilho Assumpção ${ }^{1}$
}

\begin{abstract}
Resumo: A informação é um recurso empregado pelo Estado, para controlar indivíduos ou grupos sociais. Este, que é chamado de poder informacional do Estado é evidenciado nas ações Policiais na medida em a confidencialidade, integridade e disponibilidade são requisitos para as telecomunicações nas operações de segurança pública. Para tanto, se desenvolveu um segmento de mercado de telecomunicações denominado Missão Crítica, destinado a atender estes requisitos dos órgão oficiais de segurança pública. A modalidade de telecomunicações por rádio é chamada de Professional Radio Mobile, e utiliza recursos de criptografia, modulação digital troncalizada em um processo que envolve a tecnologia, a organização e recursos humanos. Dadas as limitações desses agentes no processo, ideias de melhorias a partir das experiências de uso podem ser estimuladas, e de maneira estruturada, categorizada como informação obtida pela percepção de padrões, compor uma proposta que vise a construção de um ambiente de inovação na área de telecomunicações do órgão. Para isso, se pode fazer um estudo de caso a partir da análise de elementos préestruturais de infraestrutura (laboratórios, testes) recursos humanos (formados e capacitados em ações de compartilhamento dos aprendizados pelo uso) e fomento (recursos financeiros). Estas ações visam resultar em contribuições para a segurança da informação em operações policiais e assim, contribuir para a manutenção do poder informacional do Estado.
\end{abstract}

Palavras-Chave: Telecomunicações. Segurança Pública. Inovação.

\section{INTRODUÇÃO}

O papel das telecomunicações em uma operação policial é tão importante e decisivo quanto o armamento e viaturas utilizadas (SILVA,1998). O bom uso dos recursos de telecomunicações nas atividades de segurança pública podem representar para o Estado, o controle da informação e assim, na visão de Braman (2006) reforçar o papel do Estado na manutenção do poder informacional.

Assim é necessário que os equipamentos de comunicação estejam sempre em condições de pronto emprego, garantindo ainda a confidencialidade,

${ }^{1}$ Luciano Castilho Assumpção, mestrando do Programa de Pós-Graduação em Ciência da Informação da Universidade Federal de Santa Catarina - UFSC, orientado pelo Prof. Dr. Marcelo Minghelli. E-mail: professor.lucianoc@gmail.com. 
disponibilidade e integralidade (MORAES, 2010), das informações que circulam e que são recursos para a tomadas de decisões em ações desse tipo.

Neste sentido, um seguimento de mercado para telecomunicações de órgãos oficiais surge, chamado de Missão Critica, visando aumentar o caráter confidencial e a manutenção de quanto mais disponível, tratando a informação digitalmente. (AMARAL, 2006).

Os órgãos oficiais de segurança pública enquanto organizações e os recursos humanos também são fatores que colaboram em um processo em que a tecnologia é empregada de forma segura. Assim, propostas de melhoramentos nos processos de telecomunicações por suas experiências de uso na forma de informações estruturadas podem ser consideradas como inovação.

O presente trabalho, visa propor a construção de um ambiente de inovação dentro da área de Telecomunicações e descrever seus reflexos contribuição para a manutenção do poder informacional do Estado, dentro do órgão de segurança pública.

\section{JUSTIFICATIVA}

As telecomunicações são ferramentas estratégicas para ações de Segurança Pública evidenciadas segundo Sousa (2011), na simples observação do dia-a-dia das atividades policiais. Considerando a necessidade de comunicação de órgão oficiais, surge no mercado um segmento chamado Mission Critical ${ }^{2}$, destinado a prover radiocomunicação crítica à órgãos oficiais, utilizando-se das características de confidencialidade e integralidade oferecidas pela tecnologia. (AMARAL, 2006).

O uso frequente destes recursos em diferentes situações dentro as atribuições da Órgão de Segurança Pública geram uma série de ideias de como aproveitar melhor os recursos disponibilizados pelas ferramentas de comunicação, considerando as características de cada atividade e suas limitações.

Estes são percebidos pelos usuários do sistema de telecomunicações pelo uso frequente do equipamento. Muitas vezes não requerem novas tecnologias, ou desenvolvimento de soluções complementares ou modificações no projeto, o que certamente implicaria em grande aporte financeiro (ROSEMBERG, 2006).

\footnotetext{
2 Tecnologia de telecomunicação por meio de rádio móvel profissional, ou Professional Mobile Radio (PMR) voltado para mercados governamentais de segurança pública, conhecido como Government Radio Network (GRN)..( AMARAL, 2006).
} 
Organizar estas ideias, validando em um sistema com a infraestrutura adequada e com os recursos humanos financeiros necessários, podem ser pontos que colaborem para a reunião de condições necessárias para a criação de um ambiente formal de inovação, e assim, se beneficiar das políticas de inovação previstas em lei, refletindo de maneira direta nas políticas de uso dos equipamentos de telecomunicações em operações da Órgão de Segurança Pública.

Assim, a pesquisa se propõe a investigar fatores que contribuem em conjunto com a tecnologia empregada, e a manutenção do Estado no controle da informação o que BRAMAN (2006) chamou de Estado Informacional, por meio de inovações envolvendo recursos técnicos-organizacionais e recursos humanos.

\section{DELIMITAÇÃO DO PROBLEMA}

Conforme definido no art. 144 da Carta Magna (BRASIL, 1988), a polícia é órgão federal permanente que compõe a estrutura da Segurança Pública, sendo responsável pelas funções de polícia marítima, aeroportuária e de fronteiras e, com exclusividade, pela função de polícia judiciária da União.

Tal organização deve ainda prevenir e reprimir tráfico ilícito de entorpecentes e drogas afins, o contrabando e o descaminho; apurar as infrações penais contra a ordem política ou social ou em detrimento de bens, serviços e interesses da União ou de suas entidades autárquicas e empresas públicas, assim como outras infrações cuja prática tenha repercussão interestadual ou internacional e exija repressão uniforme.

A partir do Ano de 2005, o órgão começou a fazer uso de uma rede de telecomunicações digital, concebida para substituir uma rede analógica antiga, considerando alguns requisitos de por exemplo, a possibilidade transmitir dados, uso de protocolo aberto (tecnologias não proprietárias), sistema de localização de veículos, criptografia ponta a ponta. A tecnologia escolhida para isso foi a tetrapol. (SOUZA, 2011).

Eram previstos recursos para cerca de 100 estações de radiocomunicação, com cerca de 9000 terminais ativos até o final de 2011, segundo Souza (2011). No entanto, Cavalin (2007), já alertava para uma série de dificuldades como a falta de profissionais com mão de obra especializada em tecnologia tetrapol nos quadros da do órgão, na fase inicial da implantação, ausência de software adequado para predição em estudos de cobertura alterações constantes no projeto de rádio 
engenharia, dentre outros problemas o que hoje reflete se em problemas com relação a área de cobertura, em razão do número de estações previstas no projeto diante de uma área de atuação de $91.426 \mathrm{Km}^{2}$ segundo Souza (2011) além de problemas com a manutenção e disponibilidade do sistema (estações e terminais) em razão da falta de profissionais capacitados para a operação de rede e instruções.(CAVALIN, 2007).

Isso faz com que em algumas oportunidades, em razão destas limitações se possa lançar mão de recursos inadequados. Para minimizar este problema, diversas iniciativas são buscadas, baseado nas características de uso observadas pelos próprios usuários do sistema nos mais diversos cenários os quais estão inseridos (Amazônia Legal, patrulhamento em fronteiras, crimes financeiros, segurança de dignitários, repreensão a entorpecentes, polícia marítima, etc.) além dos profissionais inseridos na gestão da rede de telecomunicações a fim de extrair o melhor do equipamento mesmo diante dessas dificuldades.

Ocorre que estas iniciativas são realizadas de forma não estruturadas e muitas dessas inciativas acabam não sendo compartilhadas ou documentadas o que acabam sendo consideradas soluções locais e paliativas que com o passar do tempo acabam sendo esquecidas e caem em desuso novamente.

A partir deste cenário, o presente estudo tem como objetivo, analisar a possibilidade de implantar um ambiente de inovação na área de telecomunicações de órgão federal de Polícia, de modo a estruturar estas iniciativas, e contribuindo para a manutenção do poder informacional do Estado no órgão.

\section{OBJETIVO GERAL}

O objetivo desta pesquisa é analisar a possibilidade de um ambiente de inovação na área de Telecomunicações no Órgão e suas contribuições para segurança pública a partir da manutenção do poder informacional do Estado.

\section{OBJETIVOS ESPECÍFICOS}

Para auxiliar no processo de análise proposto no objetivo geral do presente estudo, consideramos os seguintes objetivos específicos:

5.1 Propor um fluxo de informação para classificar as experiências de uso em uma das dimensões de sistemas de Informação (Organizacional, Humana ou 
Tecnológica); para as propostas de inovação por experiências de aprendizagem pelo uso nos recursos de telecomunicações e descrever como elas podem contribuir para a manutenção do poder informacional nas telecomunicações do órgão de segurança pública;

5.2. Analisar as infraestruturas para validação de experiências de inovação no âmbito do sistema de telecomunicações no órgão;

5.3. Propor ações que incentivem a capacitação de usuários do sistema de telecomunicações do órgão de segurança pública; para que recursos humanos do corpo técnico e operacional se aproximem dos recursos de telecomunicações com um olhar inovador, visando extrair melhor do equipamento;

5.4 Relacionar as ações de inovação para a manutenção do poder informacional do Estado enfatizando as percepções de padrões e suas contribuições para o bom uso de equipamentos de telecomunicações nas operações de Segurança Pública.

\section{REVISÃO DE LITERATURA}

Brasil (2009) descreve o conceito de Segurança Pública como sendo a garantia da manutenção da ordem pública (situação e o estado de legalidade normal), em que as autoridades exercem suas precípuas atribuições e os cidadãos as respeitam, mediante a aplicação do Poder de Polícia, prerrogativa do Estado.

Neste sentido, evidencia-se o caráter estratégico que a informação possui para o Estado: a de exercer o poder. O poder em questão é o poder informacional, aquele que molda o comportamento humano pela manipulação da base informacional do poder instrumental, estrutural e simbólico. Tais manipulações possibilitam novas ferramentas de exercício do poder, conforme descrito por Braman (2006, p1):

Os governos contemporâneos utilizam a informação e as tecnologias de informação de nova maneira e tais práticas em seu objetivo irão mudar a natureza do poder e do seu exercício.

Dentre as abordagens do conceito de informação como elemento de sustentação do poder do Estado, segundo Braman (2006), neste estudo a trataremos inicialmente como recurso para tomada de decisões dentro de um órgão de Segurança Pública, aquela que circula na rede de telecomunicações do órgão, e em um segundo contexto; como percepção de padrões que sustentem um ambiente 
de inovação na área de telecomunicações

Órgãos oficiais de Segurança Pública tem buscado formas de se elevar o nível de segurança da informação (AMARAL, 2006). O caráter sigiloso das operações, a restrição de informações no contexto policial se faz necessário uma vez que em razão das transformações, sociais, políticas tecnológicas da sociedade que fazem com que os sistemas de informação e de telecomunicações se tornem-se instrumentos de poder para o Estado. (BRAMAN, 2006).

No primeiro contexto, na classificação de Braman (2006), a informação é considerada recursos de tomada de decisão e utilizadas como estratégia de poder informacional é necessário atentar para requisitos que colaborem com a manutenção deste poder. A afirmação de Braman (2006), sustentam a de Souza (2011) no contexto da segurança pública, já que este sustenta que pela simples observação das rotinas operacionais, pode-se comprovar que a comunicação é uma ferramenta relevante condução de missões de salvamento e segurança. O uso adequado, bem como a observação de requisitos de segurança da informação constituem em proteções a informação (ISO,2006)

Assim, de acordo com Semola (2003); as proteções necessárias precisam estar relacionadas:

a) Confidencialidade - propriedade que limita o acesso à informação tão somente as áreas legítimas;

b) Integridade - propriedade que garante que a informação manipulada mantenha todas as características originais estabelecidas pelo proprietário da informação, incluindo controle de mudanças e garantia do seu ciclo de vida e;

c) Disponibilidade - propriedade que garante que a informação esteja sempre disponível para o uso legítimo.

Shannon descreveu em 1948 um fenômeno em que a informação em transito, ou seja, partindo de uma fonte emissora de informação ela é adaptada transformando em sinais que trafegam por um meio de comunicação para que possa ser transmitida de um ponto, sendo recebida posteriormente em um destino, através de um receptor que reconheça os sinais emitidos pelo transmissor.

As fontes de ruído podem agir sobre o sinal transmitido, podendo causar alguma alteração no sinal recebido, prejudicando a sua inteligibilidade (quando não se possui sinais suficientes para que se possa compreender o conteúdo da 
informação ou integridade (quando a informação recebida é diferente da que foi transmitida).

Segundo Moraes (2010), a inconsistência da informação podem causar problemas relacionados à segurança da informação. No fluxo de informação no processo descrito por Shannon (1948), o ruído descrito no processo de pode ser ocasionado por qualquer uma das dimensões envolvidas, seja tecnológica, organizacional ou humana (LAUDON \& LAUDON, 2010).

Ainda segundo Shannon (1948), o ruído pode alterar ou impedir que a mensagem do emissor chegue ou chegue corretamente ao destinatário, pode ser considerado um fator que interfira de alguma forma na segurança da informação.

Araújo (2009), descreve na corrente teórica que envolve a matemática que Shannon(1948) chama de ruído no fluxo de informação reconhecem que as questões relativas à comunicação envolvem três níveis de problemas:

O primeiro trata dos problemas técnicos, relativos ao transporte físico da materialidade que compõe a informação. O segundo nível se refere aos problemas semântico (relacionado ao entendimento da mensagem), O terceiro nível é relaciona-se com a eficácia da comunicação (relacionado a validade da informação).

Considerando a problemática do ruído no fluxo de informação descrito por Shannon (1948), surge um segmento chamado Professional Mobile Radio (PMR) voltado para Government Radio Network (GRN) conhecido como Mission Critical, que Amaral (2006) descreve como sendo um sistema destinado a prover radiocomunicação crítica a órgãos oficiais, utilizando-se das vantagens de confidencialidade e integralidade oferecidas pelo trunking.

Segundo Amaral (2006) um sistema de missão crítica é um sistema que, se interrompido, leva a organização a um estado de crise, em um intervalo de tempo pequeno e a tecnologia de radiocomunicação digital chamada de rádio troncalizado ou (trunking $)^{3}$, contribuindo assim para a confidencialidade das comunicações, já que as mensagens são alocadas em diferentes canais de maneira randômica, dificultando ainda mais, se aliada à digitalização, codificação e criptografia, as e interceptações das mensagens, pois a estação receptora deve acompanhar as mudanças de canais de acordo com a gerência eletrônica do sistema. Assim esta

\footnotetext{
${ }^{3}$ Tecnologia de comunicação que oferece um processo em que a alocação dinâmica dos canais ocorre a cada contato entre as estações, independentemente do último canal utilizado, por meio do envio de um sinal de controle (AMARAL, 2006).
} 
tecnologia se apresenta como uma alternativa visando a garantia da integridade e confidencialidade da informação, já que a modulação e a codificação realizada no modo analógico, é mais susceptível as escutas e interceptações indesejáveis, o que implicaria no domínio do Estado, no caso o órgão de Segurança Pública, sob a informação ali tratada.

Os padrões PMR mais empregados em sistemas troncalizado digitais em Missão Crítica são Tetra, Apco 25 e Tetrapol. No órgão de Segurança Pública em estudo, o padrão empregado atualmente é o Tetrapol. (SOUZA, 2011).

Capurro (2007) entende que em uma visão sistêmica, a informação passa a ter uma corrente teórica envolvendo aspectos humanos e sociais, considerando além das questões tecnológicas, as econômicas, espaciais, ocupacionais, culturais.

Para Laudon e Laudon (2010), uma organização, participa do sistema com seus processos e com sua hierarquia, enquanto a ferramenta tecnológica deve interagir com os recursos humanos, observando as seguintes dimensões:

a) Organizações: A execução e coordenação das atividades, utilizandose da hierarquia e dos seus processos de negócio;

b) Humana: Se não existirem pessoas devidamente capacitadas para operá-los os sistemas de informação são inúteis;

c) Tecnologia: Hardware, Software, Armazenamento, Comunicação e Redes; e Infraestrutura.

Neste contexto, a manutenção do poder informacional deve ser vista como um processo, com componentes tecnológicos, organizacionais e humanos. Assim, os requisitos de integridade, confiabilidade e disponibilidade precisam ainda ser analisados sobre três dimensões (ELEUTÉRIO, 2016):

As tecnológicas que envolvem as ferramentas de tecnologia da informação e comunicações propriamente ditas, considerando seu conjunto ambiental, hardware, software, redes, etc., e todos os recursos relacionados, como criptografia, tolerância a falhas etc.

As dimensões organizacionais referem-se por sua vez a ações administrativas e de gestão que podem contribuir para os requisitos de segurança, como por exemplo, prover recursos ou condições que permitam realizar investimentos financeiros visando ampliação ou manutenção dos sistemas, além de ações que fomentem intermete o uso seguro dos recursos.

Já a dimensão humana envolve treinamento de usuários e técnicos para 
operar um sistema. Envolvem comportamentos que melhorem suas condições de segurança.

Capurro (2007) descreve a necessidade de se atentar para as dimensões além da tecnológica, sugerindo uma necessidade de que após o crescimento da dimensão tecnológica, a qual descreve como cibernética, é necessário reumanizar o conceito de informação, colocando novamente a organização e o homem como parte integrantes do processo.

Assim, pessoas podem para o melhor uso da tecnologia pela organização. Muitas vezes, pequenos melhoramentos sugeridos pelo uso de equipamentos que não o alteram diretamente, mas sugerem uma forma de usar melhor diante das necessidades e limitações de uma organização (técnica, financeira, logística, etc.). É o que Rosemberg (2006), chama de aprendizagem pelo uso. De outra forma, as experiências de usuários podem refletir a necessidades de desenvolvimento de soluções incorporadas ao produto (no caso, equipamento de telecomunicações).

Neste contexto de inovação, as informações aí geradas podem ser categorizadas como percepções de padrão (uma vez que foram observadas pelo uso frequente de um recurso) segundo Braman (2006). Nela a informação é a base para a inovação, em novas tecnologias, novos processos e movimentações nas estruturas sociais que contribuem para o poder informacional do Estado.

Damanpour (1984) define inovação como sendo a adoção de uma ideia ou de um comportamento que podem ser representados por sistemas, programas, políticas, dispositivos, processos, produtos ou serviços, não necessariamente inéditos, mas que sejam novos para a organização.

Segundo o Manual de Oslo (OCDE, 2005), inovação: "É a implementação de um produto (bem ou serviço) novo ou significativamente melhorado, ou um processo."

Mazucatto (2014), descreve uma relação com fornecedores visando a inovação como sistema de inovação parasitário. Nele, a iniciativa privada absorve as demandas de inovação geradas pelo setor público, agindo de certa forma nos processos de criação do setor privado.

$\mathrm{Na}$ definição de Maciel (1997), um ambiente de inovação refere-se ao conjunto de fatores políticos, econômicos, sociais e culturais que estimulam ou dificultam a inovação em uma organização.

Carvalho e Machado (2013) entendem que nos sistemas de inovação, os 
elementos provenientes da herança cultural e da criatividade peculiar de um determinado grupo social não são reconhecidos como componentes da inovação, diferente do que ocorre nos ambientes de inovação.

Para Minghelli (2018), a viabilidade de um ambiente de inovação, pode ser avaliada considerando as infraestruturas da organização para organização e validação das experiências de inovação; o fomento de novas práticas, treinamentos e difusão de ideias, e os recursos humanos e financeiros para tanto.

Estas inovações obtidas pelas informações a partir de percepção de padrões, em um contexto de segurança pública são relevantes a melhoria manutenção do poder informacional pela polícia de estado, considerando a informação, enquanto recurso da organização, dentro de uma análise segundo BRAMAN (2006).

\section{PROCEDIMENTOS METODOLÓGICOS}

Trata-se de pesquisa de natureza de aplicada de objetivo exploratório, em uma abordagem qualitativa sob uma perspectiva construtivista, que é empregada para buscar compreender relações (CRESWELL,2007) entre a manutenção do poder informacional e os sistemas de telecomunicações, e suas efeitos aplicados à Segurança Pública.

Seguindo o descrito por Gil (2002) uma pesquisa exploratória, tem como objetivo proporcionar maior familiaridade com o problema, com vistas a torná-lo mais explícito ou a constituir hipóteses, no caso a viabilidade de um ambiente de inovação na organização em estudo partindo das percepções de usuário.

Para tanto, seguindo o preconizado por Creswell (2007), considerando seu objetivo exploratório, foi empregada uma abordagem qualitativa através de procedimentos de pesquisa bibliográfica, de coleta de dados e análise embasada na teoria através de uma pesquisa experimental, utilizando-se a técnica de estudo de estudo de caso em um ambiente específico de órgão federal de segurança pública.

O objetivo da pesquisa bibliográfica não é realizar apenas a repetição do que já foi escrito sobre o assunto, mas através da relação proposta é proporcionar o exame dos conceitos sob uma nova abordagem. (MARCONI, 1999).

$\mathrm{Na}$ coleta de dados, o objetivo é promover observações diretas a fim de realizar levantamento, utilizando-se além das observações e entrevistas em campo, de relatórios de operações. Para Creswell (2007) a pesquisa experimental é capaz 
de identificar objetos de estudo e variáveis que possam influenciar os objetos de estudo e mecanismos de controle e observação dos efeitos que as variáveis produz no objeto em estudo, através da observação das ações dos agentes envolvidos a fim de coletar experiências de uso e seus aprendizados; com o objetivo de classificá-las sob uma dimensão, a fim de estruturar o fluxo da informação gerada, para que se possa descrição das Infraestruturas existentes e dos recursos humanos e financeiros que colaborem para a implantação de um ambiente de inovação na área de telecomunicações no órgão de Segurança Pública

Nestas fases para Gil (2002) as coletas de dados devem ser realizadas, através de procedimentos de campo, que envolvem acesso às áreas da organização ou informantes, material e informações gerais sobre procedimentos a serem desenvolvidos, a fim de obter informações qualitativas e quantitativas que ratifiquem a hipótese inicial

$\mathrm{Na}$ análise, empregando uma estratégia de teoria embasada (CRESWELL, 2007), busca-se estabelecer uma interação dos dados de diferentes grupos ( inovação, telecomunicações, segurança pública), e a partir dessa relação descrever como o cenário na organização e os elementos que podem colaborar com a implantação de um ambiente de inovação na área de telecomunicações, a partir das experiências de aprendizagem pelo uso pela percepção de padrões e como ela pode trazer reflexos para a manutenção do poder informacional do Estado para segurança pública conforme descrito por Braman (2006).

Visando atender a metodologia descrita, sugere-se a realização das etapas conforme o cronograma:

\begin{tabular}{|c|c|c|c|c|c|c|c|c|c|c|c|c|c|c|c|c|c|c|c|c|c|c|c|c|}
\hline \multirow[b]{2}{*}{ Atividade } & \multicolumn{10}{|c|}{2018} & \multicolumn{12}{|c|}{2019} & \multicolumn{2}{|c|}{2020} \\
\hline & $\sum^{\frac{\alpha}{\alpha}}$ & $\frac{\underline{\alpha}}{\frac{m}{\alpha}}$ & $\Sigma$ & 3 & 马 & 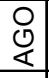 & \begin{tabular}{|l} 
\\
$\omega$ \\
\end{tabular} & 5 & $\begin{array}{l}3 \\
\text { Oे } \\
z\end{array}$ & \begin{tabular}{|c} 
U⿱山口心 \\
\end{tabular} & 孞 & 妾 & $\sum_{\Sigma}^{\frac{\alpha}{\alpha}}$ & $\frac{⿱}{\frac{\alpha}{\alpha}}$ & $\sum$ & 3 & $\stackrel{1}{3}$ & \begin{tabular}{|l|l|} 
\\
0 \\
\hdashline \\
\end{tabular} & $\stackrel{5}{\omega}$ & 5 & ○े & 㟔 & 孞 & 出 \\
\hline Coleta de referências bibliográficas & $x$ & $x$ & $x$ & $x$ & $x$ & $x$ & $x$ & & & & & & & & & & & & & & & & & \\
\hline $\begin{array}{l}\text { Participação de seminários, fóruns } \\
\text { e eventos }\end{array}$ & & & & $\mathrm{x}$ & $\mathrm{x}$ & $\mathrm{x}$ & $\mathrm{x}$ & $\mathrm{x}$ & $\mathrm{x}$ & $\mathrm{x}$ & $\mathrm{x}$ & $\mathrm{x}$ & $\mathrm{x}$ & & & & & & & & & & & \\
\hline $\begin{array}{l}\text { Atividades de coleta de dados de } \\
\text { aprendizagem pelo uso }\end{array}$ & & & & & $\mathrm{x}$ & $\mathrm{x}$ & $\mathrm{x}$ & $\mathrm{x}$ & $\mathrm{x}$ & $\mathrm{x}$ & $\mathrm{x}$ & $\mathrm{x}$ & $\mathrm{x}$ & & & & & & & & & & & \\
\hline $\begin{array}{l}\text { Atividades de coleta de dados de } \\
\text { pre-estruturas para ambiente de } \\
\text { inovação }\end{array}$ & & & & & $\mathrm{x}$ & $\mathrm{x}$ & $\mathrm{x}$ & $\mathrm{x}$ & $\mathrm{x}$ & $\mathrm{x}$ & $\mathrm{x}$ & $\mathrm{x}$ & $\mathrm{x}$ & & & & & & & & & & & \\
\hline Analises dos dados obtidos & & & & & & & & $x$ & $x$ & $x$ & $\mathrm{x}$ & $x$ & $\mathrm{x}$ & $x$ & & & & & & & & & & \\
\hline Tabulação da Pesquisa & & & & & & & & & & $\mathrm{x}$ & $\mathrm{x}$ & $x$ & $\mathrm{x}$ & $\bar{x}$ & $x$ & $x$ & $\mathrm{x}$ & & & & & & & \\
\hline $\begin{array}{l}\text { Redação do relatório parcial da } \\
\text { pesquisa }\end{array}$ & & & & & & & & & & & & & & $x$ & $x$ & $x$ & $\mathrm{x}$ & $x$ & $\mathrm{x}$ & & & & & \\
\hline $\begin{array}{l}\text { Revisão da escrita e critica final do } \\
\text { trabalho de pesquis }\end{array}$ & & & & & & & & & & & & & & & & & & $x$ & $x$ & $x$ & $x$ & & & \\
\hline Redação final da pesquisa & & & & & & & & & & & & & & & & & & & $x$ & $x$ & $x$ & $x$ & & \\
\hline Entrega final e escrita do trabalho & & & & & & & & & & & & & & & & & & & & & & & & \\
\hline
\end{tabular}




\section{CONSIDERAÇÕES PARCIAIS}

Os resultados devem refletir uma coleção de ações inovadoras baseadas em informações coletadas na percepção de padrões observados a partir das experiências dos usuários, de aprendizado pelo uso que podem contribuir para a implantação de um ambiente de inovação segundo Minghelli (2018), a partir da análise da infraestrutura para validação das iniciativas de inovação, e recursos humanos e financeiros para que elas possam ser difundidas a usuários de mesmo perfil, por meio de instruções específicas.

- Todas essas ações como o objetivo de contribuir para o processo de telecomunicações em ações de segurança pública, tendo como base a segurança da informação a por consequência a manutenção do poder informacional do Estado (BRAMAN, 2006), representado pela Órgão de Segurança Pública.

\section{REFERÊNCIAS}

AMARAL, Cristiano Torres. Interoperabilidade nos Padrões de Rádio Troncalizado Digital. Trabalho de Conclusão do Curso de Pós Graduação em Sistemas de Telecomunicações Modernos. Universidade Federal Fluminense, Belo Horizonte, 2006.

ARAUJO, Carlos Alberto Ávila. Correntes Teóricas da Ciência da Informação. Ci. Inf. Brasília, DF, v.38, n.3, p.192-204. Set/Dez, 2009.

BRASIL. Constituição (1988). Constituição da República Federativa do Brasil. Brasília, DF: Senado Federal: Centro Gráfico, 1988

BRAMAN, Sandra. Change of State. Cha Information, Policy, and Power. Ca The MIT Press. Cambridge Mand London, 2006.

CAPURRO, Rafael; HJORLAND, Bierguer. O Conceito de Ciência da Informação. Tradução de Ana Maria Pereira Cardoso, Maria da Gloria A. Ferreira, Marco Antônio de Azevedo. Perspectivas em Ciência da Informação. v.12, n.1, p.248207.Jan/Abr., 2007.

CARVALHO, L. C.; MACHADO, D.D.P.N. Ambiente de Inovação: estudo comparativo entre três unidades de uma organização do setor metal-mecânico. Revista deEmpreendedorismo e Gestão de Pequenas Empresas, v. 2, n.1, p. 4776, 2013. 
CAVALLIM, Nilson Luiz. A Implantação do Sistema Digital de Radiocomunicação TETRAPOL na Polícia Federal. 2007. Monografia (Curso Especial de Polícia) Academia Nacional de Polícia, Brasília, DF, 2007.DSIC/GSIPR.

CRESWELL, John W. Projeto de pesquisa: métodos qualitativo, quantitativo e misto.Tradução de Luciana de Oliveira da Rocha. - 2. ed. - Porto Alegre: Artmed,2007.

DAMANPOUR, F.; EVAN, W. M. Organizational innovation and performance: the problem of organizational lag. Administrative Science Quarterly, v. 29, p. 392-409, 1984.

ELEUTÉRIO, Marcos Antônio. M. Sistemas de Informações Gerenciais na Atualidade. Curitiba: Intersaberes, 2016.

GIL, Antônio Carlos. Como Elaborar Projetos de Pesquisa. São Paulo: Altas,2002. INTERNATIONAL STANDARDING ORGANIZATION. Norma ISO/IEC 27002, 2006.

LAUDON, K; LAUDON, J. Sistemas de Informação Gerenciais, 9. ed. São Paulo: Pearson Prentice Hall, 2010.

MACIEL, M. L. Inovação e conhecimento. In: SOBRAL, F. et al. (Orgs.). A alavanca de Arquimedes: ciência e tecnologia na virada do século. Brasília: Paralelo 15, 1997.

MARCONI; Maria de Andrade; LAKATOS, Eva Maria. Técnicas de Pesquisa. 4. ed. São Paulo: Atlas, 1999.

MAZZUCATO, Mariana. O estado empreendedor: desmascarando o mito do setor público vs. setor privado; tradução Elvira Serapicos. 1 a ed. São Paulo: Portfólio-Penguin, 2014.

MINGHELLI, Marcelo. Notas das aulas de Tópicos Especiais - Estado, Direito e Inovação: interfaces para a construção de ambientes de inovação. Mestrado Acadêmico em Ciência da Informação. Universidade Federal de Santa Catarina; 2018.

MORAES, Alexandre Fernandes. Redes de Computadores Fundamentos. 7 ed. São Paulo: Érica, 2010.

SEMOLA, Marcos. Gestão da Segurança da Informação. Uma visão Executiva. Editora Campus, Rio de Janeiro, 2003.

SHANNON, Claude. A Mathematical Thory of Comunication. The Bell System Technical Journal, v.27, p. 379-423, p. 623-656. Jul/Out, 1948.

SOUZA, José Luiz Povill de. REDE BRASILEIRA DE RADIOCOMUNICAÇÃO SEGURA: uma opção nacional. Monografia apresentada ao Departamento de Estudos da Escola Superior de Guerra como requisito à obtenção do diploma do Curso de Altos Estudos de Política e Estratégia Rio de Janeiro: ESG, 2011.

OCDE - MANUAL DE OSLO - Proposta de diretrizes para coleta e interpretação de dados sobre inovação tecnológica. (2005). Disponível em: http://download.finep.gov.br/imprensa/manual_de_oslo.pdf. Acesso em 25 de maio de 2018.

ROSENBERG, N. Por dentro da caixa-preta: tecnologia e economia. Campinas: 
Editora da Unicamp, 2006.

SILVA, Edmilson Nunes da. Manual de Radiocomunicação. Brasília-DF, ANP, 1998. 